\title{
Combined model of non-conformal layer growth for accurate optical simulation of thin-film silicon solar cells
}

\author{
Martin Sever a,*, Benjamin Lipovšek ${ }^{\mathrm{a}}$, Janez Krč ${ }^{\mathrm{a}}$, Andrej Čampa ${ }^{\mathrm{a}}$, Guillermo Sánchez \\ Plaza $^{\mathrm{b}}$, Franz-Josef Haug ${ }^{\mathrm{c}}$, Martial Duchamp ${ }^{\mathrm{d}}$, Wim Soppe ${ }^{\mathrm{e}}$, Marko Topič ${ }^{\mathrm{a}}$ \\ a University of Ljubljana, Faculty of Electrical Engineering Tržaška cesta 25, Ljubljana 1000, Slovenia \\ b Technical University of Valencia, Valencia Nanophotonics Technology Center (NTC) Valencia 46022, Spain \\ ${ }^{\mathrm{c}}$ Ecole Polytechnique Federale de Lausanne (EPFL), Institute of Microengineering (IMT), Photovoltaics and Thin-Film Electronics Laboratory, Neuchatel 2000, \\ Switzerland \\ ${ }^{\mathrm{d}}$ Ernst Ruska-Centre for Microscopy and Spectroscopy with Electrons Institute for Microstructure Research, Research Centre Jülich, Jülich D-52425, Germany \\ e ECN-Solliance, High Tech Campus 5, Eindhoven 5656 AE, The Netherlands
}

\section{A R T I C L E I N F O}

Available online 30 May 2013

Keywords:

Thin-film silicon solar cells

Non-conformal growth

Textured interfaces

Light management

Rigorous optical modelling

\begin{abstract}
A B S T R A C T
In thin-film silicon solar cells textured interfaces are introduced, leading to improved antireflection and light trapping capabilities of the devices. Thin-layers are deposited on surface-textured substrates or superstrates and the texture is translated to internal interfaces. For accurate optical modelling of the thin-film silicon solar cells it is important to define and include the morphology of textured interfaces as realistic as possible. In this paper we present a model of thin-layer growth on textured surfaces which combines two growth principles: conformal and isotropic one. With the model we can predict the morphology of subsequent internal interfaces in thin-film silicon solar cells based on the known morphology of the substrate or superstrate. Calibration of the model for different materials grown under certain conditions is done on various cross-sectional scanning electron microscopy images of realistic devices. Advantages over existing growth modelling approaches are demonstrated-one of them is the ability of the model to predict and omit the textures with high possibility of defective regions formation inside the Si absorber layers. The developed model of layer growth is used in rigorous 3-D optical simulations employing the COMSOL simulator. A sinusoidal texture of the substrate is optimised for the case of a micromorph silicon solar cell. More than a $50 \%$ increase in short-circuit current density of the bottom cell with respect to the flat case is predicted, considering the defect-free absorber layers. The developed approach enables accurate prediction and powerful design of current-matched top and bottom cell.
\end{abstract}

(c) 2013 Elsevier B.V. All rights reserved.

\section{Introduction}

In R\&D of high-efficiency thin-film silicon solar cells, light management plays a substantial role [1,2]. Introducing a proper surface texture to the substrate or superstrate of a solar cell can result in enhanced light scattering and anti-reflection effect, improving light confinement in the cell. This can lead to increased photocurrent and consequently higher conversion efficiency if electrical properties of the cell remain unaffected.

Besides random textures of transparent conductive oxides (such as $\mathrm{SnO}_{2}: \mathrm{F}$ [3], low pressure chemical vapour depositionLP-CVD of ZnO:B [4], or magnetron sputtered ZnO:Al [5]) and nano-textured silver back contacts [6], artificial periodic textures

\footnotetext{
* Corresponding author. Tel.: +38614768723.

E-mail address: martin.sever@fe.uni-lj.si (M. Sever).
}

have also gained much interest, showing the potential to match or even surpass the light trapping capabilities of random textures [7]. By using interference lithography, UV nano-imprint lithography, electroforming process for fabrication of large shims and UV embossing process, large area substrates with desired textures can be made on industrial scale [8-10].

In the design of efficient textures, two- or three-dimensional rigorous optical modelling is an essential tool [11-22]. For accurate determination of the optimal texture parameters realistic parameters of a solar cell have to be considered. Besides the optical properties of the layers (wavelength dependent complex refractive indices) and their thicknesses, the exact morphologies of all the textured interfaces within the device also need to be taken into account. In many publications on rigorous optical modelling of thin-film solar cells, fully conformal layer growth is considered (e. g. $[14,15,18,23])$ by assuming that the initial texture morphology of the substrate or superstrate is ideally transferred to all the other 
internal interfaces within the thin-film structure. However, realistic cross-sectional images of thin-film solar cells reveal that this is usually not the case $[24,25]$. Recently we indicated that if thicker layers are introduced or textures with high aspect ratio (ratio of vertical to lateral surface feature sizes) are used, the conformal approximation is not sufficient anymore and a combination of different growth mechanisms is required [26]. Some new approaches to the modelling of non-conformal layer growth have been researched and reported recently. These address specifically the growth of microcrystalline layers [27], or growth in general, by rounding of rectangular textures at subsequent interfaces [20], or applying one type of growth, for example, perpendicularly to the surface normal [28].

In this paper we present a combined 3-D model of nonconformal layer growth in which not only one, but a combination of two growth principles are implemented: a conformal and an isotropic one. We demonstrate on different realistic examples that the developed model, although simple and easy for implementation in optical simulations, matches with experimental the crosssectional images of realistic structures very well and outperforms the existing single-type growth models. After calibration and verification, the growth model is integrated in rigorous 3-D optical simulations of thin-film silicon solar cells employing the COMSOL simulator, which uses the finite element method (FEM) for solving Maxwell's equations. Using the optical simulations, the sinusoidal surface texture of the substrate of a micromorph $(a-\mathrm{Si}: \mathrm{H} / \mu c-\mathrm{Si}: \mathrm{H})$ solar cell is optimised, with respect to increased photocurrent and, at the same time, assuring crack-free absorber layers in the device. The model enables to predict and omit the sharp valleys in the resulting textures that can lead to detrimental crack formations in semiconductor layers [27]. Using COMSOL simulations, the external quantum efficiency and short-circuit current of the micromorph device are calculated and the optimal period and height of the sinusoidal substrate texture are determined, considering all mentioned aspects.

\section{Experimental}

Masters with periodic textures were fabricated on silicon wafers using e-beam lithography and reactive ion etching. As a master with random texture standard Asahi $\mathrm{U} \mathrm{SnO}_{2}: \mathrm{F}$ transparent conductive oxide on the glass substrate was used. Ultraviolet nanoimprint lithography was employed to replicate masters on the solar cell substrates covered with a lacquer [29,30]. Amorphous solar cells were deposited using VHF-PECVD at $70 \mathrm{MHz}$ with substrate temperature $T_{\text {sub }}=190{ }^{\circ} \mathrm{C}$, dilution $\mathrm{H}_{2} / \mathrm{SiH}_{4}=2$ and pressure $0.4 \mathrm{mbar}$. Microcrystalline cells were deposited by RF-PECVD, at $T_{\text {sub }}=200{ }^{\circ} \mathrm{C}$ using $200 \mathrm{sccm} \mathrm{H}_{2}$ and 3.5-5 sccm $\mathrm{SiH}_{4}$. Supporting layers: $\mathrm{Ag}, \mathrm{ZnO}$ and ITO were magnetron sputtered (DC for Ag and RF for $\mathrm{ZnO}$ and ITO) in all types of cells. Cross-sectional images of solar cell structures were done with Hitachi IM4000 and FEI helios nanolab $400 \mathrm{~s}$ scanning electron microscope (SEM) equipped with a focus ion beam facility.

\section{Modelling}

\subsection{Model of non-conformal layer growth}

When building realistic optical models one of the important issues is to fit the geometry of the modelled structure to the actual structure of a solar cell as well as possible. In the case of thin-film solar cells, special attention has to be paid to the realistic representation of interfacial nano- and micro-textures in multilayer structures, since they determine the light scattering and antireflection properties. Here we present an empirical model of layer growth which combines two growth principles: (i) conformal and (ii) isotropic. In the first principle, the morphology of the interfaces of the grown layers remains unchanged (Fig. 1a), it is simply vertically transferred from the initial substrate to the surface of the growing layer. This can be modelled by applying the growth in vertical direction of the structure only. The second type of growth principle (isotropic) describes the growth in the direction of the normal vector at any given point on the surface (Fig. 1b). Isotropy in this case refers to equal growth in all directions from each discrete point on the surface, where the resulting envelope presents the surface. Independently of the deposition method during fabrication (CVD, PVD, and condensation), a proper combination of these two types of growth was shown to result in good representation of the interface morphologies after thin-film layer depositions, except when the grown layer produces additional large texture features during growth (e.g. a thick LP-CVD ZnO or hot Ag layer). This was confirmed by the cross-sectional SEM images of various thin-film silicon solar cell structures (single junction, tandem with amorphous and microcrystalline silicon, metal layers, flat transparent conductive layers) grown on either random or periodic textures. In our model this ratio between the two growth rates (isotropic over conformal) is set by the empirical parameter called the growth parameter $(g)$.
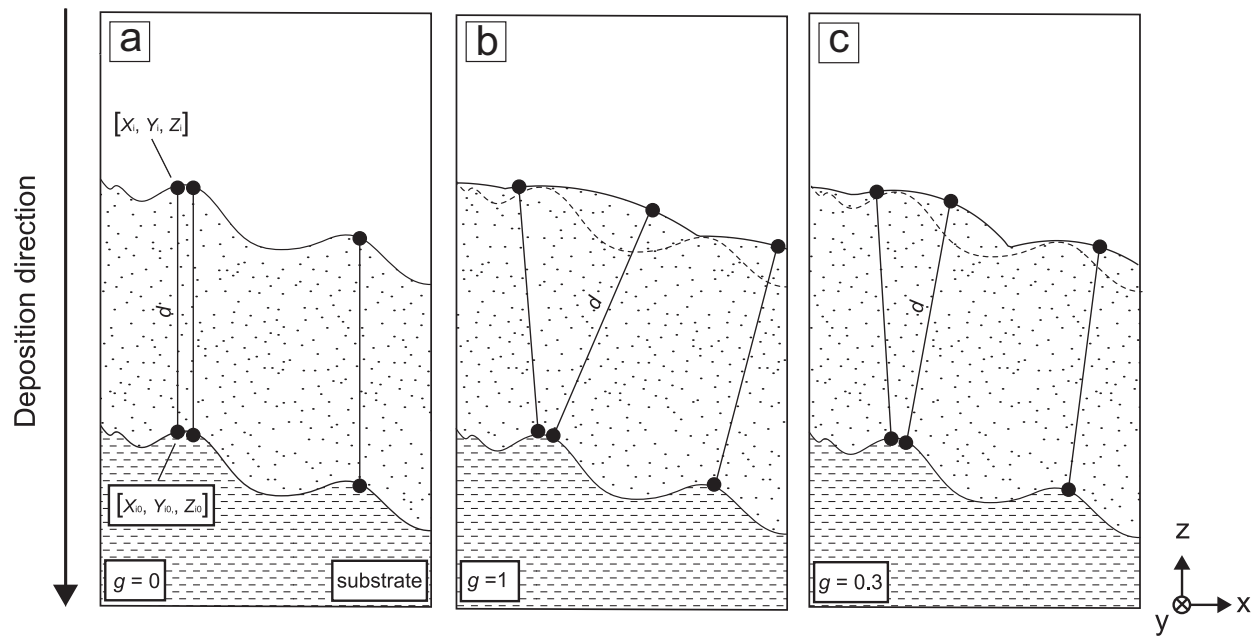

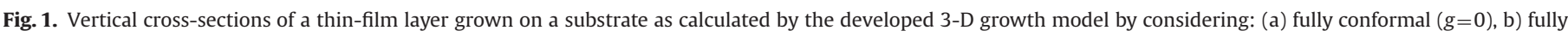

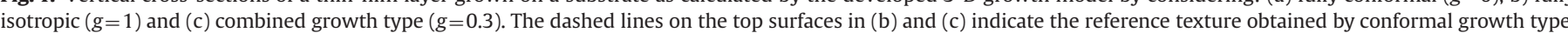


It determines the resulting direction of the combined growth, while the length of the growth (distance between the initial point and the point on the new surface) is assigned to $d$, which we refer to as the layer thickness. For completely conformal growth when $g$ equals 0 (see example in Fig. 1a) and for solely isotropic when growth $g$ equals 1 (Fig. $1 \mathrm{~b}$ ). The values between 0 and 1 define a linear combination of the two growth rates. Fig. 1c shows an example of a growth corresponding to $g=0.3$. For the sake of simplicity of representation, Fig. $1(\mathrm{a}-\mathrm{c})$ only shows vertical crosssections of the structures, whereas the developed model is fully three dimensional and thus considers both lateral $(x, y)$ and a vertical dimension $(z)$.

Using atomic force microscopy (AFM), the morphology of the initial surface texture-either the substrate or the top surface of the previously deposited layer-can be determined. This presents the input data for (initial texture) for our model. Each discrete point (i) on the surface is described by its lateral position $x_{i 0}, y_{i 0}$ and the vertical position $z_{i 0}$ (relative height). Subscript 0 refers to the initial surface. From multiple neighbouring points in both lateral dimensions, locally averaged unit normal vector of the surface is calculated in the $i$ th point and labelled as shown in Eq. (1):

$\vec{n}_{l}=\left[\begin{array}{c}n_{x i} \\ n_{y i} \\ n_{z i}\end{array}\right], \quad\left|\vec{n}_{l}\right|=1$

In order to apply the correct layer thickness $(d)$ to the grown layer, which we consider to be equal to the thickness of the layer grown on a flat surface under the same conditions, we need to define a scaling factor $s_{\mathrm{i}}$ (Eq. (2)). The $s_{\mathrm{i}}$ ensures, that every pair of points $i_{0}$ and $i$ is exactly $d$ apart.

$s_{i}=\sqrt{\left(n_{x i} g d\right)^{2}+\left(n_{y i} g d\right)^{2}+\left(n_{z i} g d+(1-g) d\right)^{2}}$

After calculating $n_{i}$ and $s_{i}$ for each discrete point on the surface, the corresponding new points, defining the morphology of the next interface, can be calculated as:

$\left[\begin{array}{l}x_{i} \\ y_{i} \\ z_{i}\end{array}\right]=\left[\begin{array}{l}x_{i 0} \\ y_{i 0} \\ z_{i 0}\end{array}\right]+\frac{g d}{s_{i}}\left[\begin{array}{l}n_{x i} \\ n_{y i} \\ n_{z i}\end{array}\right]+\frac{(1-g) d}{s_{i}}\left[\begin{array}{l}0 \\ 0 \\ 1\end{array}\right]$

The second and third part of Eq. (3) correspond to the isotropic and conformal contribution to the growth respectively.

Due to the nature of calculations, some of these points may form internal loops in the material (e.g. as shown later in Fig. 6c) and must therefore be discarded. Only the highest point at a certain lateral position has to be considered. However, we observe that regions where internal points are overlapped in the model coincidence with the locations at SEM images where defective regions (cracks) are identified (discussed later). On the other hand there are regions of fewer points and they require interpolation.

An important property of the model is that it follows the physical law of superposition of growths. The resulting texture from a single step growth from initial texture is the same as if the growth is separated in several, partial growths of a layer. With multiple steps we can also get more information about the defective regions at a certain position inside a layer (not only situation at final surface).

Before application of the model into rigorous optical simulations of realistic solar cells, it needs to be calibrated-one must adjust the growth parameter $(g)$ for each layer grown under particular deposition or sputtering conditions on the selected testing textures. Cross-sections obtained by scanning or transmission electron microscopy (SEM, TEM) of realistic structures are used for this purpose. The adjustment of the $g$ can be automated by minimisation of the root-mean-square error between the surface morphology of the realistic device and the modelled device on a few samples. Once $g$ parameters are determined for each layer, the calibrated model can be utilised to predict interface morphology for new textures, where (i) lateral and vertical dimensions of initial surface morphology are changed (optimised) or (ii) thicknesses of layers are varied.

Besides predicting the morphologies of interfaces for optical simulations, another important role of the proposed growth model is to predict sharp angles (V-shapes) in the morphology of the grown layers (Fig. 2). It has been shown that sharp valleys often lead to the formation of defective regions-cracks, in silicon layers (amorphous and especially microcrystalline), significantly deteriorating the electrical properties of a solar cell [7,27,31].

Experimental results indicated that in the case of substrate configuration of thin-film silicon solar cells this opening angle should be greater than $135^{\circ}$ to avoid the formation of defective regions in microcrystalline and amorphous silicon layers [8,32,33]. With the model we can also detect and omit textures with sharp valleys, which would cause crack formation in the layers. Thus, not only optical aspects (high photocurrents) but also electrical ones, i.e. preserving or even improving the fill factor and open-circuit voltage of the device, can be considered in optical simulations. All of these considerations are crucial in the design of the surface textures for high-efficiency solar cells [34-37].

\subsection{COMSOL simulator}

In our 3-D optical simulations, the COMSOL Multiphysics simulation software [38] was employed. Simulated 3-D structures with all interfaces were imported from the calibrated growth model; discretisation for optical simulations was done inside the COMSOL. Rigorous solving of Maxwell's equations is based on the finite element method (FEM). COMSOL's CAD-like graphical interface enables simple implementations of different geometries of 3-D multi-layer structures, such as thin-film solar cells with either periodic or random interface morphologies. In the case of structures with lateral periodicity and symmetrical properties, integrated symmetry boundary conditions enable simulation over one quarter of a structure period only, reducing the time of simulation significantly. COMSOL enables dynamic adjustment of the meshing depending on the wavelength of light. Since meshing in FEM is not restricted to rectangular elements, these structures can be modelled with great geometrical precision. This is important for thin-film solar cells where various nano-textures (periodic and random) are studied for efficient light trapping. The FEM method requires separate simulations for each wavelength of light. Many integrated iterative and direct methods for solving the

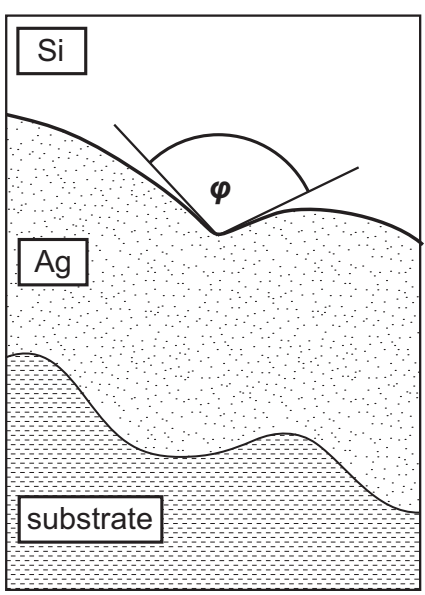

Fig. 2. V-shape valley formed after layer growth (example of Ag layer, simulation of growth) is characterised by the opening angle $\varphi$. The V-shape valley is not present at the initial substrate surface but is a consequence of the layer growth. 
system of equations are available and applicable to specific solar cell structures. Recently, methods enabling inclusion of thick layers (such as glass) in FEM simulations of thin-film solar cells were reported as well [39].

\section{Results}

\subsection{Application and verification of the growth model}

As the effect of applying three different $g$ parameters on the resulting morphology of a grown layer was indicated in Fig. 1, here we show the effect of applying different layer thicknesses in the model. The $g$ parameter was chosen to be 0.4 in this case. Results are presented in Fig. 3. We can see that by increasing the thickness, the layer surface becomes smoother. The initial substrate texture (Fig. 3a) in this case is a real AFM scan of a randomly textured LP-CVD ZnO $(2.5 \mu \mathrm{m}$ thick). The root-mean-square roughness of the surfaces $\left(\sigma_{\mathrm{RMS}}\right)$ corresponding to different layer thicknesses $(d=0 \mathrm{~nm}$, $200 \mathrm{~nm}, 500 \mathrm{~nm}$ and $1000 \mathrm{~nm}$ ) decreases with the thickness of growth as specified here: (a) $86.9 \mathrm{~nm}$ (roughness of the substrate), (b) $82.4 \mathrm{~nm}$, (c) $77.6 \mathrm{~nm}$ and (d) $74.0 \mathrm{~nm}$. Thus, the model also predicts the decrease in roughness as a function of layer thickness, which presents an important information for 1-D scalar scattering models $[40,41]$ as well.

In next section we apply the growth model on realistic thinfilm silicon solar cells. We demonstrate that both growth principles are necessary for accurate modelling of the layer growth. In Fig. 4a we show an example where periodically textured substrate with sinusoidal texturing was used in a single-junction microcrystalline thin-film $(\mu c-\mathrm{Si}: \mathrm{H})$ silicon solar cell in substrate configuration. In Fig. 4b the results of the modelling are presented with white curves. For the sake of simplicity we show them only for the $\mathrm{ZnO} / \mathrm{n}-\mu c-\mathrm{Si}: \mathrm{H}$ interface (middle curve) and for the the $\mathrm{p}-\mu c-\mathrm{Si}: \mathrm{H} /$ $\mathrm{ZnO}$ interface (top curve). The bottom curve represents the substrate morphology and its input parameter. From the crosssections, we determine the calibrated $g$ parameters to be 0.2 for $\mathrm{Ag}$
$(200 \mathrm{~nm})$ and $\mathrm{ZnO}(80 \mathrm{~nm})$ and 0.3 for the $\mu \mathrm{c}$-Si:H (800 nm) layers. Parameter $g$ is to be independent of the layer thickness. However, for its accurate determination, it is desirable to have samples with different thicknesses of the same layer for the calibration. In Fig. 4 one can see that the top surface of the $\mu c$-Si:H layer stack differs significantly from the one on the substrate, which excludes the conformal growth approach. With the developed model a good matching can be obtained. No additional texturing due to $\mu c-\mathrm{Si}: \mathrm{H}$ material needed to be considered.

The importance of using the combined model is clearly shown in Fig. 5, where a special sample with a variable period was fabricated (using e-beam process at the Valencia Nanooptics Technology Centre). In this sample, the period of the sinusoidal shape was gradually changed, obtaining different aspect ratios of the texture on one sample. In this case, single-junction amorphous silicon $(a-\mathrm{Si}: \mathrm{H})$ solar cell was deposited on this texture. The calibrated $g$ parameters corresponding to different layers were again 0.2 for $\mathrm{Ag}$ and $\mathrm{ZnO}$, whereas for $a-\mathrm{Si}: \mathrm{H}$ layers the calibrated $g$ value was 0.3

Fairly good agreement between the calculated and experimental growth is observed for the combined model (thick full lines). For comparison the results are also shown for the case of a) conformal ( $g=0$, dotted line) and b) isotropic ( $g=1$, dashed line) growth type. Root-mean-square errors between the exact morphology and the calculated ones for the top $a$-Si:H interface (top curves in the figure) are $71 \mathrm{~nm}, 120 \mathrm{~nm}$ and $35 \mathrm{~nm}$ for the conformal, isotropic and combined model, respectively.

Random textures consist of features of different sizes and orientation and are therefore good testing example for our model. Fig. 6a shows cross-section of a $\mu c-\mathrm{Si}: \mathrm{H}$ cell deposited on a randomly textured substrate. Again, parameter $g$ was calibrated to 0.2 for $\mathrm{Ag}$ and $\mathrm{ZnO}$ layer and 0.3 for $\mu \mathrm{c}$-Si:H layers. One has to point out that small rotations of cross-sectional images have to be applied in some cases to match the actual normal direction of the sample during deposition (not necessarily equal to the normal direction of SEM image). This is especially important for layers where the conformal component of growth predominates
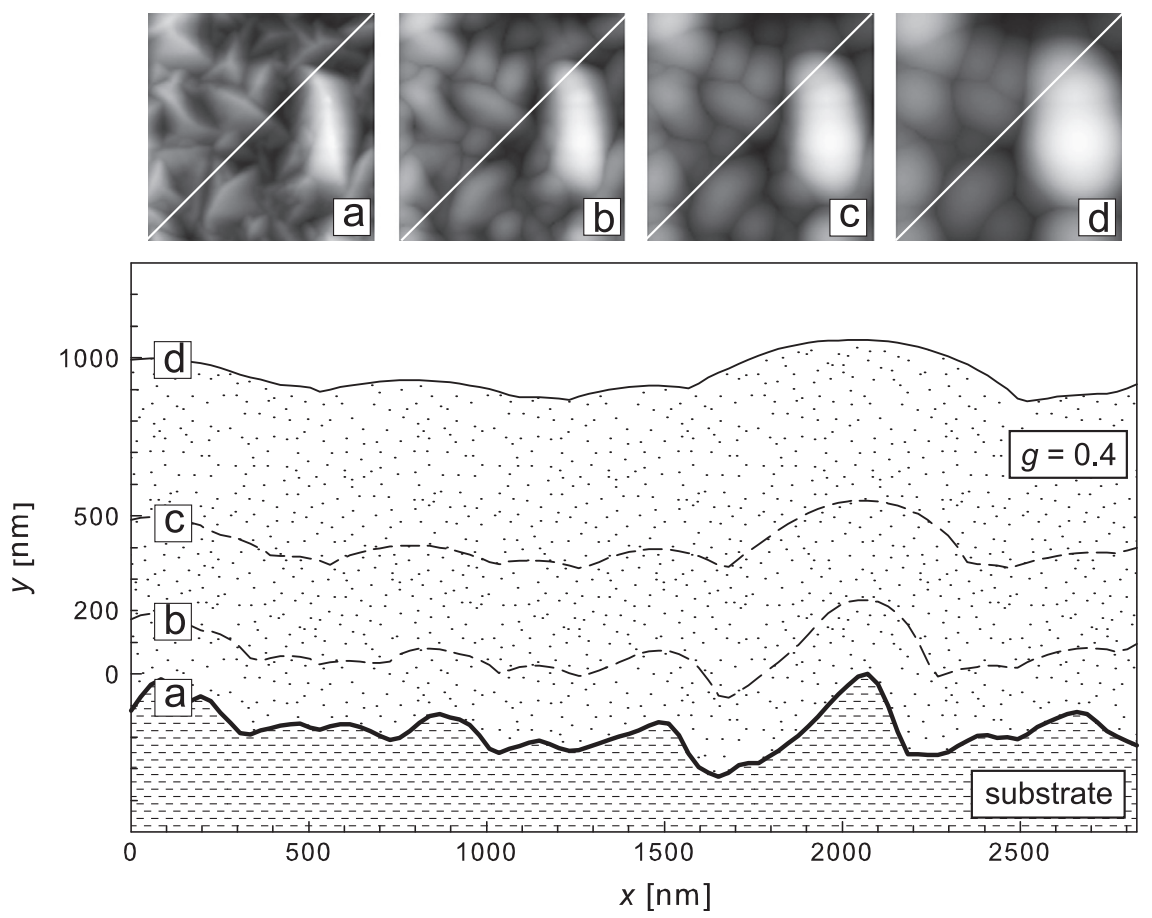



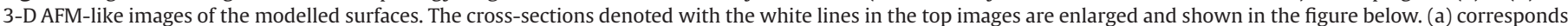
to AFM scan of the surface, whereas (b), (c) and (d) are results of calculations with the proposed model. 

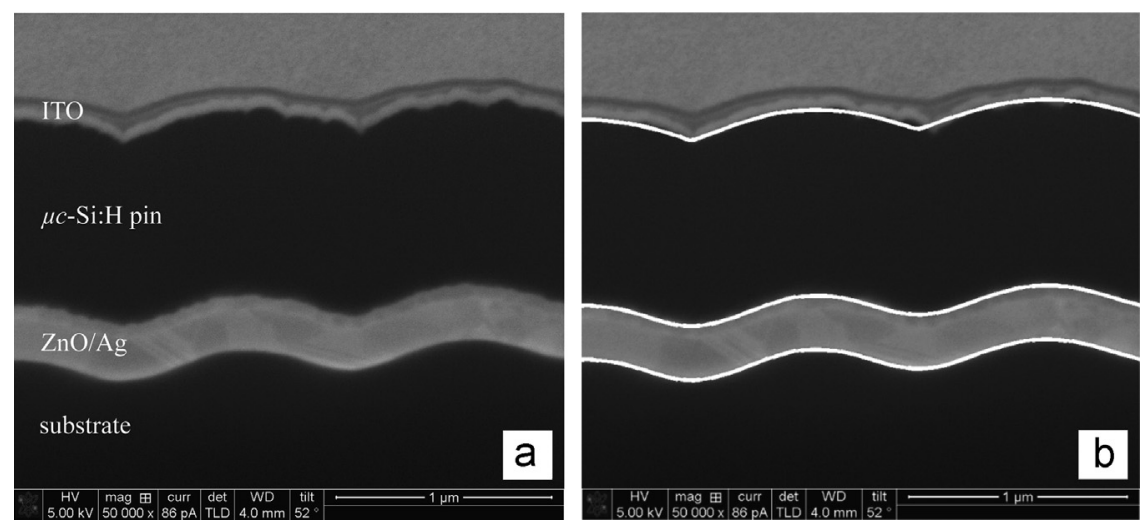

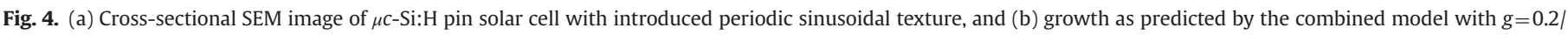
0.3 for $\mathrm{Ag}$ and $\mathrm{ZnO} / \mu \mathrm{c}$-Si:H stack (white lines).
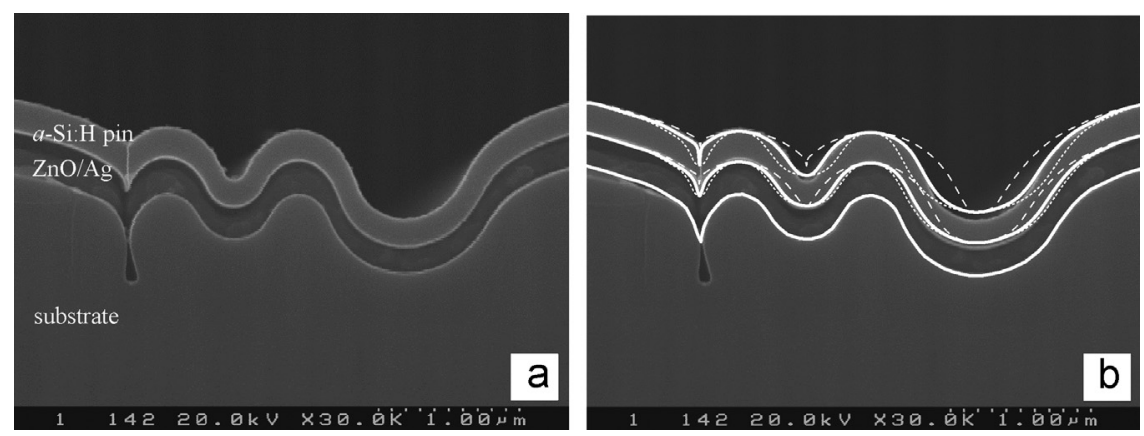

Fig. 5. (a) Cross-sectional SEM image of $a$-Si:H solar cell grown on variable sinusoidal textures, and (b) predicted growth roughness as obtained with the model, depending on the considered growth type, are shown in dotted, dashed and full lines for conformal, isotropic and combined growth type (with $g=0.2$ for Ag and $\mathrm{ZnO}$ and $g=0.3$ for $a$ Si:H layers).
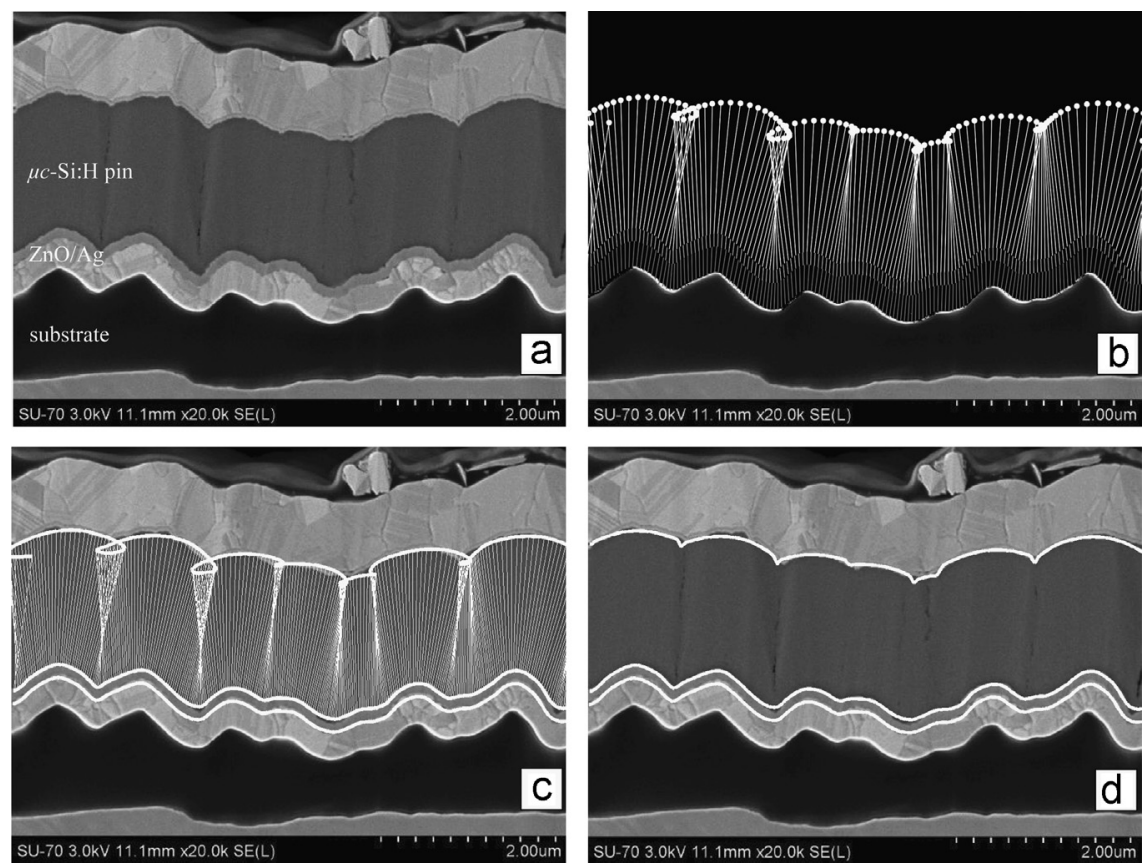

Fig. 6. (a) Cross-sectional SEM image of $\mu c$-Si:H solar cell deposited on a randomly textured substrate, (b) first step of modelling of growth—calculation of direction vectors with the corresponding final points, (c) generation of lines from the final points, (d) the highest points selection/ elimination of the loops underneath. Fig (b), (c), and (d) are results calculated by the model.

( small $\mathrm{g}$ ). The procedure of the calculation in the model is shown here in several steps. In Fig. 6b, we show calculated the directional growth vectors of combined growth with the corresponding final points for the case of top surface of the $\mu \mathrm{c}$-Si:H pin stack. In Fig. $6 \mathrm{c}$ the final points for every layer are connected to the surface representing lines. Overlapping of directional vectors, resulting in loops of points underneath the top surface of $\mu c$-Si:H layers, can be observed. The loops present in Fig. $6 \mathrm{c}$ need to be eliminated 
and, therefore, the resulting textures are presented in Fig. 6d. The interface representing lines match the real interfaces well. Furthermore, comparison of Fig. $6 c$ to Fig. 6a reveals that the regions where the directional vectors intensively overlap correspond to dark areas in Fig. 6a, indicating defective regions inside the $\mu c-\mathrm{Si}: \mathrm{H}$ material. This indicates that the model may be used to predict such regions and, by optimising the initial substrate texture, to avoid them. In this way the electrical properties of the device can be maintained or even improved.

\subsection{Optical simulation of micromorph solar cells using the improved model of layer growth}

The micromorph solar cell simulated in COMSOL is shown in Fig. 7a. Two dimensional sinusoidal texture was applied to the substrate surface and its lateral and vertical parameters were optimised. For the optical simulations, a model of the structure, including the morphology of all the interfaces, is imported from the developed model of non-conformal layer growth presented in previous sections. Realistic complex refractive indices of layers were used [40]. Fig. 7b shows an example of the meshing obtained with finite element discretisation. In the simulations, only one quarter of the structure is used due to the symmetry of the sinusoidal texture considered here. Special attention was paid to define a proper mesh in each layer, i.e., that the nano-textures are accurately described and that the distance between two points is a few times smaller than the effective wavelength of light inside the specific layer. Several trials were done to be sure that the results are not affected by the meshing.

In the structure, the thicknesses of $\mathrm{i}-a-\mathrm{Si}: \mathrm{H}$ and $\mathrm{i}-\mu c-\mathrm{Si}: \mathrm{H}$ absorber layers were fixed to $200 \mathrm{~nm}$ and $1.2 \mu \mathrm{m}$, respectively. 2-D sinusoidal texture was chosen as: (i) it is smooth in comparison to other shapes (e.g. triangular and rectangular) which already by itself reduces possibilities of forming defective regions within solar cell and (ii) previous 2-D simulations reveal high potential of sinusoidal textures for improved light trapping $[16,42,43]$. The textures of internal surfaces in the cell were changed according to the resulted morphologies from the growth model presented in the previous sections. Due to simplicity reason here the same $g$ value $(g=0.3$ ) was used for $\mathrm{Ag}, \mathrm{ZnO}, a-\mathrm{Si}: \mathrm{H}$ and $\mu c$-Si:H materials. No significant differences in optical simulation trends were detected if $g=0.2$ was used for thin $\mathrm{Ag}$ and $\mathrm{ZnO}$ layer for these textures (tested for selected $P$ and $h$ combinations, not shown here). As the top contact LP-CVD B-doped ZnO was used.
The texture of the top contact consist of randomly distributed pyramids [44], which are superimposed to the textures resulting from the growth of the underlying layers. In the approximation of the top contact texture, we consider periodically distributed pyramids with the period $(P)$ corresponding to an average correlation length of $200 \mathrm{~nm}$ and the height $(h)$ corresponding to an average peak-to-peak value of $120 \mathrm{~nm}$ [45].

In order to demonstrate the importance of using the presented model of layer growth we show a comparison of simulated external quantum efficiencies of the top $a-\mathrm{Si}: \mathrm{H}\left(E Q E_{\text {top }}\right)$ and the bottom $\mu c$-Si:H ( $\left.E Q E_{\text {bot }}\right)$ cell, obtained with different models of layer growth (Fig. 9). Here, external quantum efficiencies are equalled to the simulated absorptances in i-layers as holds very well for most state-of-the-art devices [46,47].

Fig. 8 shows that the use of different models of layer growth has a significant influence on the results of optical simulations.

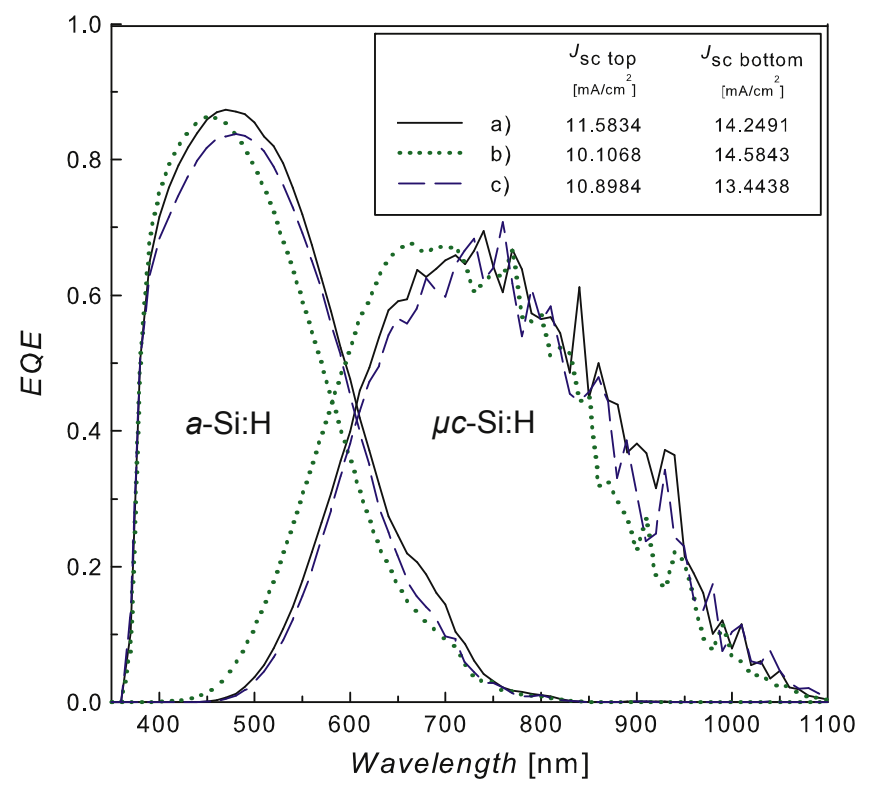

Fig. 8. Simulated external quantum efficiency of the micromorph structures obtained considering: (a) combined growth model $(g=0.3)$, (b) conformal $(g=0)$ and (c) isotropic $(g=1)$ growth. The 2-D sinusoidal texture has the period of $1100 \mathrm{~nm}$ and height of $1050 \mathrm{~nm}$ in this case.
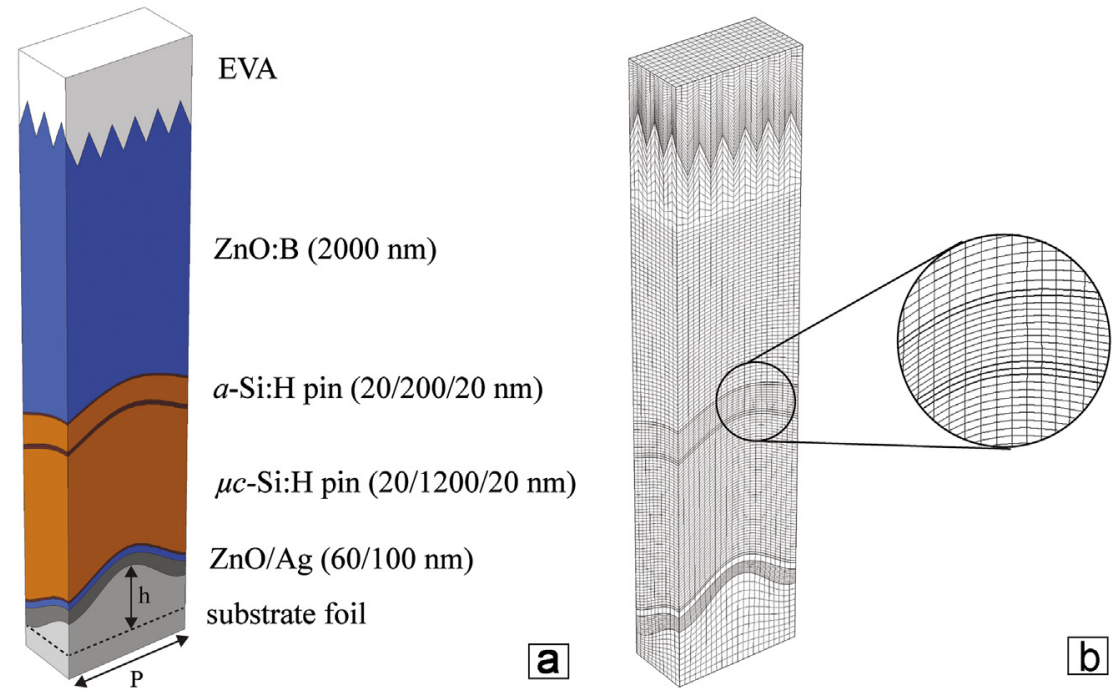

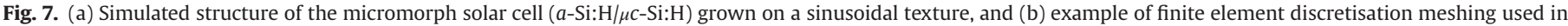
simulations. 
The differences are more pronounced if aggressive textures (with high aspect ratios) are used. In Fig. 8, there are differences in both $E Q E_{\text {top }}$ and $E Q E_{\text {bot }}$. The highest deviations are observed for conformal model (b). In particular lower $E Q E_{\text {top }}$ and higher $E Q E_{\text {bot }}$ in the wavelength range of $500 \mathrm{~nm}-700 \mathrm{~nm}$ are observed. This is a consequence of less efficient light trapping in the top absorber layer for these textures. The results of the isotropic model (c) are closer to the results of the combined model (a), but still deviate. The differences in EQEs are reflected also in the corresponding short-circuit currents $\left(J_{\mathrm{SC}}\right)$ of the top and the bottom cell given in the figure. $J_{\mathrm{SC}} \mathrm{S}$ were calculated from the absorptance in the intrinsic layers by applying the AM 1.5 spectrum. Contributions from the $p$ and $n$ doped layers were neglected as in the case of $E Q E$ calculation. $J_{\mathrm{SC}}$ values of the simulated cell (Fig. 8) reveal that the device was not current-matched. However, we can see that using different models of layer growth the ratio of top over bottom cell current is changed. Taking into account the actual nonconformal change of morphology at the interfaces of the micromorph Si solar cell this approach enables for the first time an accurate design of current-matched top and bottom cell. Furthermore, simulations also revealed that different growth models can lead to different results of texture optimisation (different $P$ and $h$ values, not shown here).

In the following we show the results of 3-D optical simulations and optimisation of the 2-D sinusoidal periodic surface texture of the substrate in the above described micromorph solar cell. The textures of all the internal interfaces were defined based on the combined model of layer growth using $g$ values as mentioned above.

Tested periods (see definition of $P$ in Fig. 7a) of the substrate texture range from $200 \mathrm{~nm}$ to $1400 \mathrm{~nm}$ in $100 \mathrm{~nm}$ increments and the heights $(h)$ from $150 \mathrm{~nm}$ to $1050 \mathrm{~nm}$ in $150 \mathrm{~nm}$ increments.

In Fig. 9 we show simulated $J_{\mathrm{SC}}$ of the top $(a-\mathrm{Si}: \mathrm{H})$ and the bottom $(\mu c-\mathrm{Si}: \mathrm{H})$ cell as a function of the period and height of the substrate texture (see legend). At this point, we refer to the thin lines in the graphs, while the thick ones (on top of thin) will be explained later on. On the left scale the absolute $J_{\mathrm{SC}}$ values are given whereas on the right scale the relative increase of $J_{S C}$ in respect to a perfectly flat, non-textured solar cell is presented.

Since this solar cell is made in substrate configuration, the top solar cell is less influenced by the already severely smoothed textures (due to the realistic growth). This is the reason, that smaller changes in $J_{\mathrm{SC}}$ as a function of $P$ and $h$ are observed for the top cell (Fig. 9a) than for the bottom cell (Fig. 9b), where over 70\% improvements are observed if proper periods and heights are selected.



As mentioned before, the growth of thin layers in thin-film solar cells can lead to formation of narrow-angle valleys even if the initial texture of the substrate is smooth. In this case we pay special attention to the top surface of the Ag contact, which appears to be critical. In order to avoid defective regions formation in subsequent $\mu c$-Si:H and $a-\mathrm{Si}: \mathrm{H}$ layers we apply the before mentioned criterion of the minimal opening angle $\left(\varphi>135^{\circ}\right)$. Selections of textures $(P$ and $h$ combinations) that fulfil this criterion were done based on the growth model and the corresponding $J_{\mathrm{SC}} \mathrm{S}$ are shown by thick lines in Fig. 9. One can observe that even if the texture of the initial substrate is sinusoidal (relatively smooth), many of $P$ and $h$ combinations do not fulfil the condition. These textures could lead to improvements in absorption and consequently $J_{\mathrm{SC}}$, but would deteriorate the fill factor of the cell [27]. Better choices for the optimal textures present the ones considering the mentioned condition. In particular the sinusoidal texture of $P=1100 \mathrm{~nm}$ and $h=300 \mathrm{~nm}$ presents the optimum according to the simulations. More than a $50 \%$ increase in $J_{\mathrm{SC}}$ bot $(+53.4 \%)$, compared to the flat cell is achieved. In the top cell, however, improvement of $J_{\mathrm{SC}}$ top is only $3.8 \%$. Since the $J_{\mathrm{SC}}$ of the top cell is not improved so much, incorporation of an intermediate reflector is of interest.

\section{Conclusions}

A model of non-conformal layer growth developed for the accurate determination of realistic morphologies of the internal interfaces in thin-film silicon solar cells was presented. The model combines conformal and isotropic growth principles, while the ratio between the two is determined by the growth parameter $g$ (per certain material and deposition conditions). The model was calibrated on crosssectional images of realistic thin-film solar cells. The importance of the model was shown by means of reduction of the mismatch between the realistic and modelled top surfaces and by displaying the significant differences in simulated $E Q E$ when different layer growth approximations are employed.

The calibrated model of layer growth was used for accurate 3-D optical simulations of micromorph $a-\mathrm{Si}: \mathrm{H} / \mu c-\mathrm{Si}: \mathrm{H}$ solar cells on sinusoidal textured substrate. The texture was optimised with respect to the highest short-circuit current of the top and bottom cell. Additionally, the layer growth model was utilised to indicate textures which could result in formation of defective regions inside the $\mathrm{Si}$ absorber layers. Such textures were excluded from the final

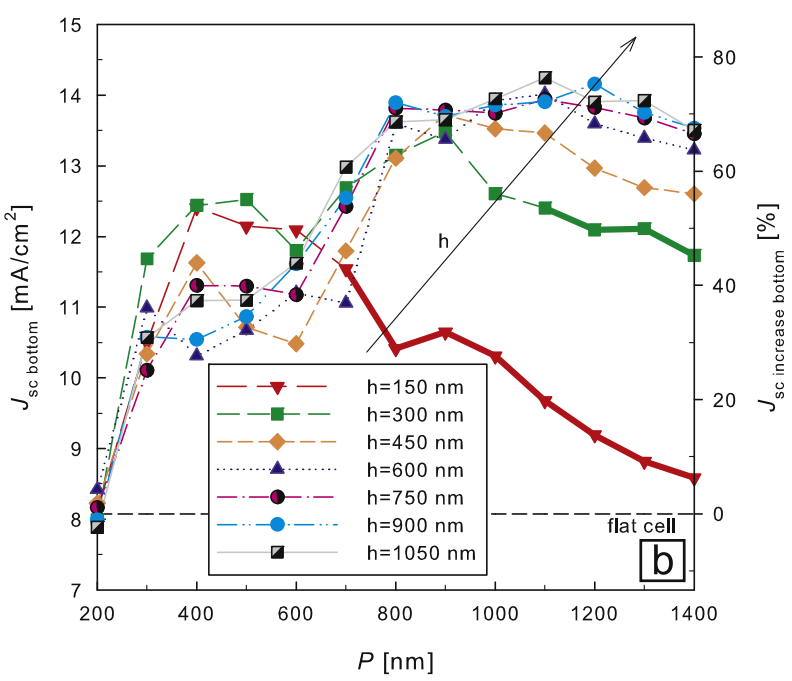

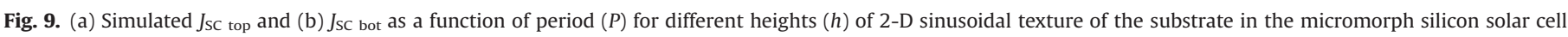
shown in Fig. 7. Thick lines correspond to validity of angular restriction of $\varphi>135^{\circ}$ at the $\mathrm{ZnO} / \mu c-\mathrm{Si}: \mathrm{H}$ interface. 
optimisation, assuring that high values of fill factor and open circuit voltage are retained for the optimised cells as well. Considering this additional condition, the optimal sinusoidal texture of the substrate was determined by the period of $1100 \mathrm{~nm}$ and height of $300 \mathrm{~nm}$ which resulted in the predicted $53.4 \% J_{\mathrm{SC}}$ increase of the bottom solar cell and $3.8 \% J_{\mathrm{SC}}$ increase of top solar cell.

\section{Acknowledgements}

The authors acknowledge the financial support from the European FP7 projects Silicon-Light (GA no. 2412777), the Integrated Infrastructure Initiative (312483-ESTEEM2) and the Slovenian Research Agency (P2-0197). The authors thank Christophe Ballif and Rémi Biron from IMT EPFL, Neuchatel and Rafal E. Dunin-Borkowski from Institute for Microstructure Research, Research Centre Jülich for their precious support to this work. M. Sever personally acknowledges the Slovenian Research Agency for providing PhD funding.

\section{References}

[1] A. Shah, Thin-Film Silicon Solar Cells, EPFL Press, 2010.

[2] J. Krč, M. Topič, Optical Modeling and Simulation of Thin-Film Photovoltaic Devices, CRC Press, 2013.

[3] K. Sato, Y. Gotoh, Y. Wakayama, Y. Hayashi, K. Adachi, H. Nishimura, Reports of the Research Labs, Asahi Glass Co., 1992.

[4] S. Faÿ, J. Steinhauser, N. Oliveira, E. Vallat-Sauvain, C. Ballif, Opto-electronic properties of rough LP-CVD ZnO:B for use as TCO in thin-film silicon solar cells, Thin Solid Films 515 (2007) 8558-8561.

[5] O. Kluth, B. Rech, L. Houben, S. Wieder, G. Schope, C. Beneking, et al., Texture etched ZnO: Al coated glass substrates for silicon based thin film solar cells, Thin Solid Films. 351 (1999) 247-253.

[6] K. Soederstroem, F.-J. Haug, J. Escarre, C. Pahud, R. Biron, C. Ballif, Highly reflective nanotextured sputtered silver back reflector for flexible highefficiency n-i-p thin-film silicon solar cells, Solar Energy Materials and Solar Cells 95 (2011) 3585-3591.

[7] C. Battaglia, C.-M. Hsu, K. Söderström, J. Escarré, F.-J. Haug, M. Charrière, et al., Light trapping in solar cells: can periodic beat random? ACS Nano 6 (2012) 2790-2797.

[8] P. Couty, M. Duchamp, K. Söderström, B.A. Kovács, R.E. Dunin-Borkowski, L. Sansonnens, et al., Transmission electron microscopy of amorphous tandem thin-film silicon modules produced by a roll-to-roll process on plastic foil, WIP, Hamburg, Germany2395-2398.

[9] W.J. Soppe, H. Borg, B.B. Van Aken, C. Devilee, M. Dörenkämper, M. Goris, et al., Roll to roll fabrication of thin film silicon solar cells on nano-textured substrates, Journal of Nanoscience and Nanotechnology 11 (2011) 10604-10609.

[10] W.J. Soppe, M. Dörenkämper, J.B. Notta, P.P.A.C. Pex, W. Schipper, R. Wilde, Nano Imprint Lithography of Textures for Light Trapping in Thin Film Silicon Solar Cells, WIP, Frankfurt, Germany2117-2119.

[11] C. Haase, H. Stiebig, Optical properties of thin-film silicon solar cells with grating couplers, Progress in Photovoltaics: Research and Applications 14 (2006) 629-641.

[12] C. Haase, H. Stiebig, Thin-film silicon solar cells with efficient periodic light trapping texture, Applied Physics Letters 91 (2007) 061116-061116-3.

[13] A. Čampa, J. Krč, F. Smole, M. Topič, Potential of diffraction gratings for implementation as a metal back reflector in thin-film silicon solar cells, Thin Solid Films 516 (2008) 6963-6967.

[14] O. Isabella, A. Čampa, W.J. Soppe, A.J.M. van Erven, R.H. Franken, et al., Diffraction Gratings for Light Trapping in Thin-Film Silicon Solar Cells, Valencia, Spain2320-2324

[15] A. Čampa, J. Krč, M. Topič, Analysis and optimisation of microcrystalline silicon solar cells with periodic sinusoidal textured interfaces by two-dimensional optical simulations, Journal of Applied Physics 105 (2009) 083107-083107-5.

[16] B. Lipovšek, M. Cvek, A. Čampa, J. Krč, M. Topič, in: Proceedings of analysis and optimisation of periodic interface textures in thin-film silicon solar cells, 25th European Photovoltaic Solar Energy Conference and Exhibition/5th World Conference on Photovoltaic Energy Conversion. (2010) pp. 3120-3123

[17] J. Chen, Q. Wang, H. Li, Microstructured design for light trapping in thin-film silicon solar cells, Optical Engineering 49 (2010) 088001-088001-9.

[18] R. Dewan, I. Vasilev, V. Jovanov, D. Knipp, Optical enhancement and losses of pyramid textured thin-film silicon solar cells, Journal of Applied Physics 110 (2011) 013101-03101-10.

[19] J. Lacombe, O. Sergeev, K. Chakanga, K. von Maydell, C. Agert, Three dimensional optical modeling of amorphous silicon thin film solar cells using the finite-difference time-domain method including real randomly surface topographies, Journal of Applied Physics 110 (2011) 023102-023102-6.
[20] S. Solntsev, M. Zeman, Optical modeling of thin-film silicon solar cells with submicron periodic gratings and nonconformal layers, Energy Procedia 10 (2011) 308-312.

[21] C. Jandl, K. Hertel, C. Pflaum, Simulation of thin-film silicon solar cells with integrated AFM scans for oblique incident waves, 2663-2666.

[22] O. Isabella, S. Solntsev, D. Caratelli, M. Zeman, 3-D optical modeling of thin film silicon solar cells on diffraction gratings, Progress in Photovoltic: Research Applications (2012) 94-108.

[23] C. Haase, H. Stiebig, Thin-film silicon solar cells with efficient periodic light trapping texture, Applied Physics Letters 91 (2007) 061116.

[24] H. Sai, K. Saito, N. Hozuki, M. Kondo, Relationship between the cell thickness and the optimum period of textured back reflectors in thin-film microcrystalline silicon solar cells, Applied Physics Letters 102 (2013) 053509-053509-5.

[25] Y. Nasuno, M. Kondo, A. Matsuda, Effects of Substrate Surface Morphology on Microcrystalline Silicon Solar Cells, Japanese Journal of Applied Physics 40 (2001) L303-L305.

[26] M. Sever, B. Lipovšek, J. Krč, M. Topič, Optimisation of surface textures in thinfilm silicon solar cells with $3 \mathrm{~d}$ optical modelling by considering realistic laye growth, in: EU PVSEC Proceedings, Frankfurt, Germany, 2012: pp. 2129-2131.

[27] M. Python, O. Madani, D. Dominé, F. Meillaud, E. Vallat-Sauvain, C. Ballif, Influence of the substrate geometrical parameters on microcrystalline silicon growth for thin-film solar cells, Solar Energy Materials and Solar Cells 93 (2009) 1714-1720.

[28] V. Jovanov, X. Xu, S. Shrestha, M. Schulte, J. Hüpkes, M. Zeman, et al., Influence of interface morphologies on amorphous silicon thin film solar cells prepared on randomly textured substrates, Solar Energy Materials and Solar Cells 112 (2013) 182-189.

[29] C. Battaglia, J. Escarre, K. Soederstroem, M. Charriere, M. Despeisse, F.-J. Haug, et al., Nanomoulding of transparent zinc oxide electrodes for efficient ligh trapping in solar cells, Nature Photonics 5 (2011) 535-538.

[30] W. Soppe, M. Dörenkämper, J.-B. Notta, P. Pex, W. Schipper, R. Wilde, Nanoimprint lithography of textures for light trapping in thin film silicon solar cells, Physica Status Solidi A 210 (2013) 707-710.

[31] M. Duchamp, M. Lachmann, C.B. Boothroyd, A. Kovács, F.-J. Haug, C. Ballif, et al., Compositional study of defects in microcrystalline silicon solar cells using spectral decomposition in the scanning transmission electron microscope, Applied Physics Letters 102 (2013) 133902-133902-3.

[32] M. Duchamp, K. Söderström, Q. Jeangros, C.B. Boothroyd, A. Kovács, T. Kasama, et al., Transmission electron microscopy of the textured silver back reflector of a thin-film silicon solar cells from chrystallography to optical absorption, WIP, Hamburg, Germany2554-2557.

[33] H.B.T. Li, R.H. Franken, J.K. Rath, R.E.I. Schropp, Structural defects caused by a rough substrate and their influence on the performance of hydrogenated nano-crystalline silicon n-i-p solar cells, Solar Energy Materials and Solar Cells 93 (2009) 338-349.

[34] F.-J. Haug, T. Söderström, M. Python, V. Terrazzoni-Daudrix, X. Niquille, C. Ballif, Development of micromorph tandem solar cells on flexible lowcost plastic substrates, Solar Energy Materials and Solar Cells 93 (2009) 884-887.

[35] H. Sai, Y. Kanamori, M. Kondo, Flattened light-scattering substrate in thin film silicon solar cells for improved infrared response, Applied Physics Letters 98 (2011) 113502-113502-3.

[36] K. Söderström, G. Bugnon, R. Biron, C. Pahud, F. Meillaud, F.-J. Haug, et al., Thin-film silicon triple-junction solar cell with $12.5 \%$ stable efficiency on innovative flat light-scattering substrate, Journal of Applied Physics 112 (2012) 114503-114503-4.

[37] R. Biron, S. Hänni, M. Boccard, C. Pahud, K. Söderström, M. Duchamp, et al., New progress in the fabrication of $n-i-p$ micromorph solar cells for opaque substrates, Solar Energy Materials and Solar Cells 114 (2013) 147-155.

[38] 〈http://www.comsol.com/〉, Multiphysics Modeling and Simulation Software, 2012.

[39] A. Čampa, J. Krč, M. Topič, Two Approaches for Incoherent Propagation of Light in Rigorous Numerical Simulations 137 (2013) 187-202.

[40] J. Springer, A. Poruba, M. Vanecek, Improved three-dimensional optical model for thin-film silicon solar cells, Journal of Applied Physics 96 (2004) 5329-5337.

[41] J. Krč, M. Zeman, O. Kluth, E. Smole, M. Topič, Effect of surface roughness of $\mathrm{ZnO}$ : Al films on light scattering in hydrogenated amorphous silicon solar cells RID A-5194-2008, Thin Solid Films 426 (2003) 296-304.

[42] O. Isabella, F. Moll, J. Krč, M. Zeman, Modulated surface textures using zincoxide films for solar cells applications, Physica Status Solidi A 207 (2010) 642-646.

[43] D. Domine, F.-J. Haug, C. Battaglia, C. Ballif, Modeling of light scattering from micro- and nanotextured surfaces, Journal of Applied Physics 107 (2010) 044504-044504-8.

[44] S. Fay, J. Steinhauser, N. Oliveira, E. Vallat-Sauvain, C. Ballif, Opto-electronic properties of rough LP-CVD ZnO: B for use as TCO in thin-film silicon solar cells, Thin Solid Films 515 (2007) 8558-8561.

[45] F. Haug, C. Battaglia, D. Domine, C. Ballif, Light scattering at nano-textured surfaces in thin film silicon solar cells, 2010: pp. 000754-000759.

[46] M. Zeman, R.A.C.M.M. van Swaaij, J.W. Metselaar, R.E.I. Schropp, Optical modeling of a-Si:H solar cells with rough interfaces: Effect of back contact and interface roughness, Journal of Applied Physics. 88 (2000) 6436-6443.

[47] J. Krč, M. Zeman, F. Smole, M. Topič, Optical modeling of a-Si: H solar cells deposited on textured glass/SnO2 substrates RID A-5194-2008, Journal of Applied Physics 92 (2002) 749-755. 